\title{
New breakthrough system announced
}

Going beyond a new implant design, Nobel Biocare is redefining the market with its N1 implant system.

The total solution sees site preparation improve with the introduction of the

OsseoShaper, a new innovative alternative to conventional drilling protocols. Experience gained from over 18 months of clinical evaluations with an early ambassador group on the Nobel Biocare N1 concept have already indicated promising results in terms of the method's effectiveness.

Fully embracing the new Mucointegration concept, the Xeal and TiUltra surfaces will be available on the Nobel Biocare N1 implant system from

\section{Summer savings!}

All Meisinger products are now available for professionals to buy direct in the UK through their sister company, myplant.

As a special offer for readers of the British Dental Journal, myplant is offering a buy one get one free deal on some of the most popular products. These include any diamond instruments, tungsten carbide instruments, ceramic abrasives, flexible polishers and steel instruments!

This is an excellent opportunity to pick up your favourite instruments at a fantastic price or even discover something new.

To find out more, contact the team at myplant on 08007797879 or email order@myplant-dental.com.

\section{Polish unrestricted}

Cord dragging has become a thing of the past with the new Proxeo TWIST Cordless from W\&H. This portable handpiece is a revelation, especially if domiciliary work is performed. It can be rotated 360 degrees within the oral cavity, enabling you to work in a comfortable, ergonomic position as you polish.

Combined with W\&H's innovative disposable prophy angles, the Proxeo TWIST Cordless is lighter than many smartphones. This minimises wrist strain and fatigue, as clinicians are able to manoeuvre the handpiece for improved access and an unrestricted view of the treatment area. day one. With the help of their specially tailored surface chemistry and topography, the Nobel Biocare N1 TiUltra implants and Xeal abutments can thus benefit from optimised tissue integration.

Nobel Biocare N1 will be further integrated into the digital workflow with DTX Studio, giving dental professionals more opportunities to provide patients with shorter-time-teeth.

Nobel Biocare N1 will first become available to clinicians in CE markets later in 2019, followed by additional markets pending regulatory approval.

More information about Nobel Biocare $\mathrm{N} 1$ is available at www.nobelbiocare.com/n1.

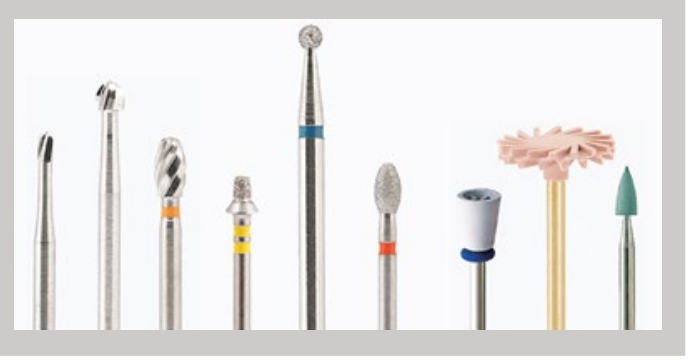

\section{A GENIUS addition}

Oral-B continues to be at the forefront

of intelligent health and beauty by introducing a new generation of brushing through its most advanced dental device to date, the NEW GENIUS $\mathrm{X}$ with Artificial Intelligence (AI).

Research from the number one brand recommended by dentists in the UK reveals $80 \%$ of people miss an area of their mouth when brushing their teeth which if not improved, could lead to serious future dental issues. The brand's smartest toothbrush yet uses AI technology to track where you are actually brushing (and not brushing enough) in your mouth to generate personalised feedback via the Oral-B app and show you how to achieve your best results every day.

By identifying those areas you've missed and helping to change your brushing habits for the better, GENIUS $\mathrm{X}$ aims to save precious time and money when it comes to dental care in the long run. Over time, the app provides guidance on how to improve your brushing habits, coaching you to the best level of care for a healthy, happy smile.

The NEW Oral-B GENIUS X with Artificial Intelligence is available in two stunning colours; a sleek black or metallic rose gold design. The new smart brush also features a modern, sleek travel case that charges both the brush and a USB device, such as a smartphone, to make travelling with an electric toothbrush easier than ever.

For further information visit: www. oralb.co.uk/en-gb/product-collections/ genius-X. speed of the handpiece can also be adjusted to suit your clinical requirements.

Find out more by visiting www.wh.com/ en_uk or call 01727874990 or email office. uk@wh.com.

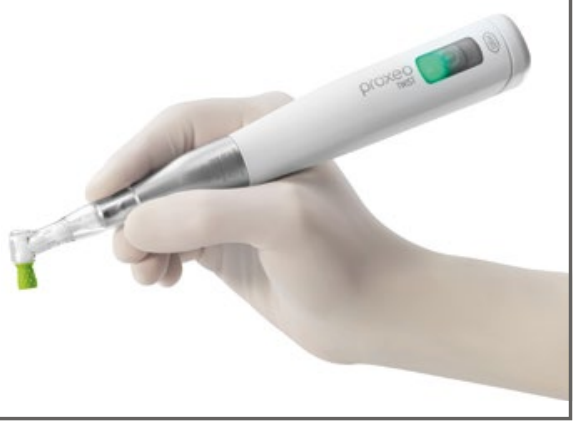

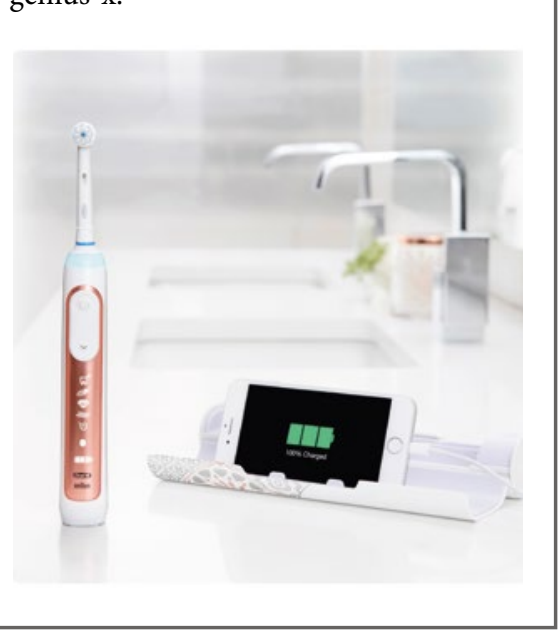

